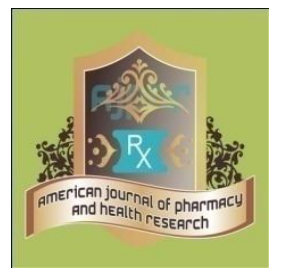

Research Article AMERICAN JOURNAL OF PHARMACY AND HEALTH RESEARCH www.ajphr.com 2018, Volume 6, Issue 08 ISSN: 2321-3647(online)

\title{
Jejunostomy Following Complete Jejunal Transection After Blunt Abdominal Trauma: A Rare Case Report
}

\author{
Sitaram yadav ${ }^{1}$, Anju Nagar ${ }^{2}$, Radheshyam Meena $^{2}$, Ramesh Suthar $^{3}$ \\ Govt. medical college Kota, Rajasthan , 324001
}

\begin{abstract}
Abdominal injury following road traffic accident is less common, compared to the extremities, head and chest. Bowel may get injured following blunt abdominal trauma, but perforation and complete transection is rare. Reports of intestinal perforation during low energy impacts such as bicycle falls, are extremely rare. We report a case of 30-year-old male, who presented with severe abdominal pain, following bicycle road traffic accident. Chest X-Ray was normal, X ray flat plain abdomen showed air under both dome of the diaphragm and abdominal FAST scan revealed intra-peritoneal free fluid with internal echoes. Early exploration and prompt surgical intervention led to the successful outcome in this patient
\end{abstract}

Keywords: Blunt trauma abdomen, isolated jejunal transection, double barrel jejunostomy. 


\section{INTRODUCTION}

A 30-year-old male was referred to our Emergency Surgical Department with severe abdominal pain and recurrent vomiting after a bicycle accident. He started vomiting approximately four hours after initial trauma, which was greenish in colour and non-projectile. There was no history of loss of consciousness, Ear, Nose and Throat (ENT) bleed, haematemesis or melaena.

On admission, 36 hours post trauma, patient was febrile and he looked toxic with signs of dehydration. Physical examination revealed pulse-120 beats/min and blood pressure $110 / 70 \mathrm{mmHg}$.

No bruises or other external injuries were noted. Abdominal examination revealed distension of abdomen with tenderness and guarding all over the abdomen. Shifting dullness was present and bowel sounds were absent.

Routine blood investigations were done which were within normal limits. Erect X-Ray abdomen showed gas under both the dome of the diaphragm [figure 1]

Abdominal FAST scan revealed intra-peritoneal free fluid with internal echoes. Computed Tomography (CT) abdomen was not done due to frank clinical diagnosis and considering the duration of trauma.

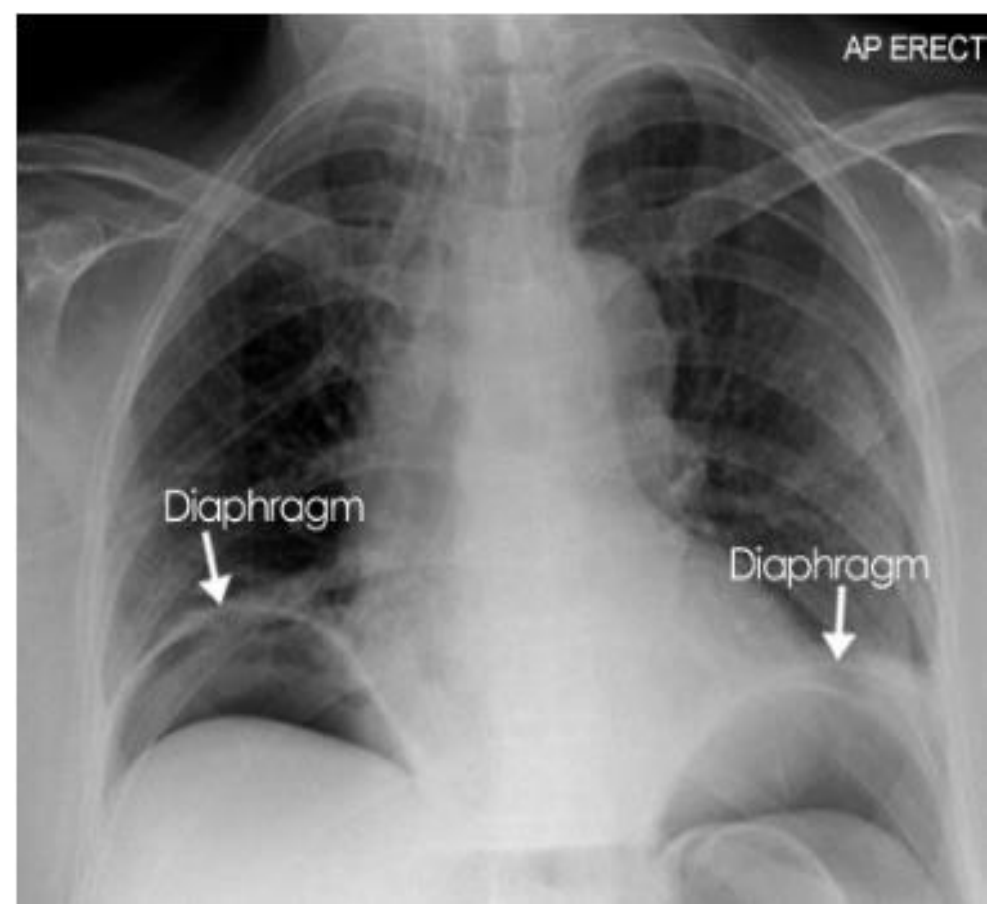

Figure 1: Erect $x$ ray showing air under both dome of the diaphragm

Patient was planned for emergency explorative laparotomy. Midline incision was taken. On exploration abdomen was found to be filled with bilious fluid. There was a complete transection of jejunum approximately $20 \mathrm{~cm}$ distal to duodenojeunal flexure.( fig 2,3 ) 

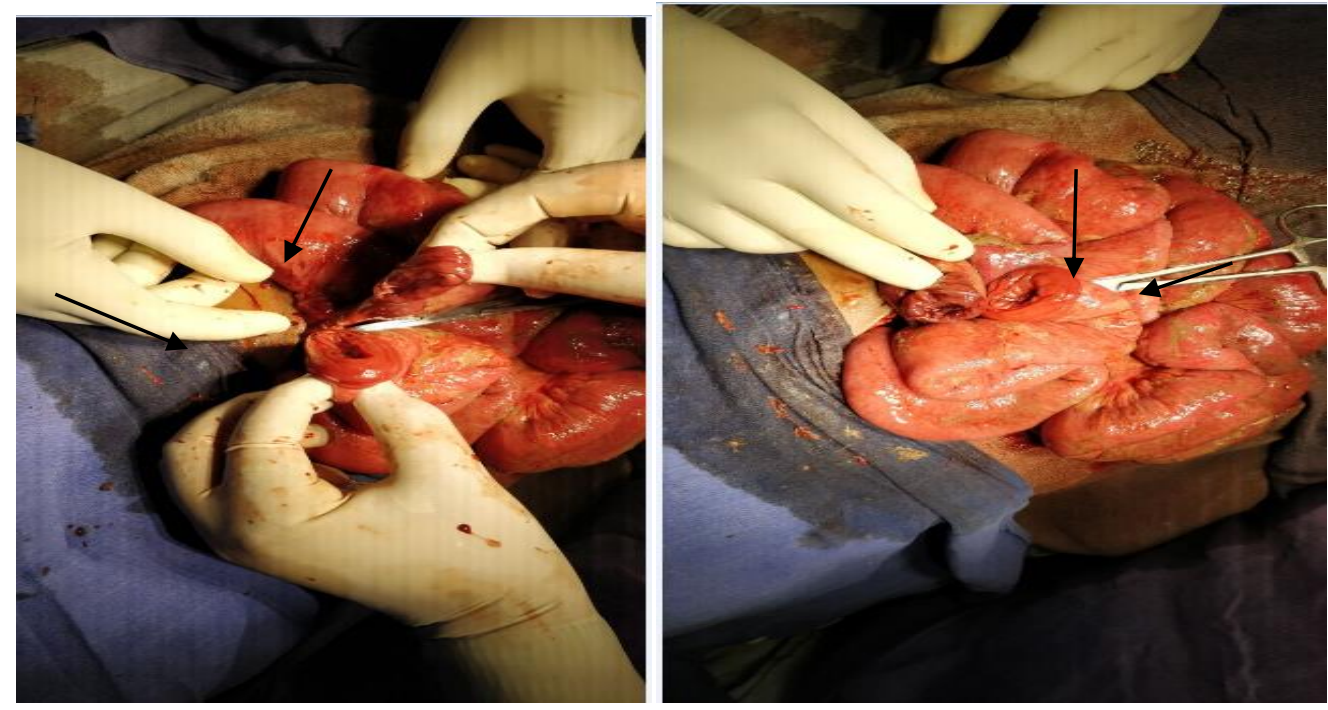

Figure 2, 3 Complete isolated transected ends of jejunum

Approximately 2 litre of bilious intraperitoneal free fluid was drained and double barrel jejunostomy was performed. Patient was managed postoperatively in intensive care unit and was discharged 2 weeks later after unremarkable postoperative recovery and patient was readmitted 40 days later for closure of double barrel jejunostomy(fig 4$)$.

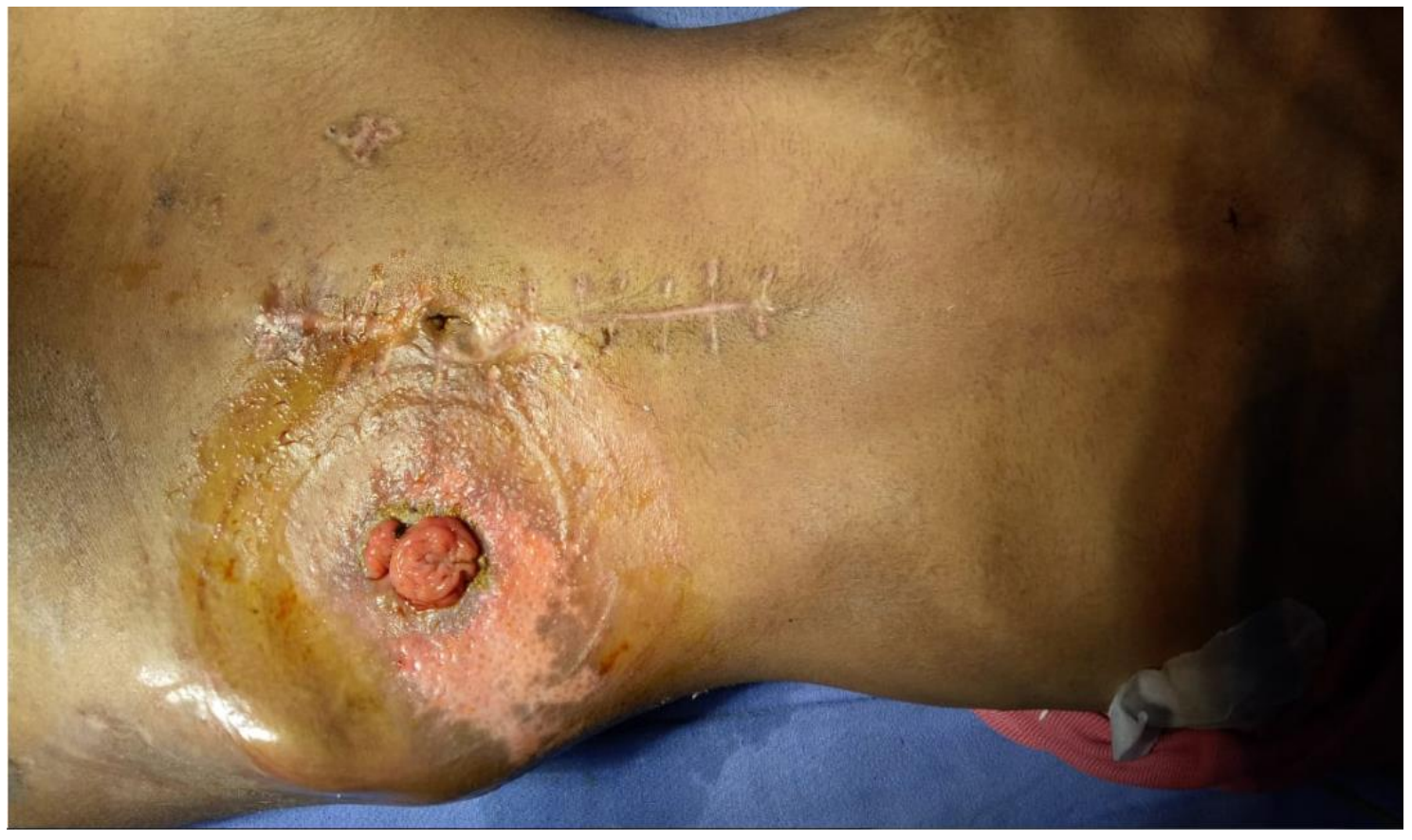

Figure 4 showing double barrel jejunostomy

Double barrel jejunostomy was closed in a single layer(fig 5) and patient was managed in postoperative ward with total parenteral nutrition for 5 days and then was discharged unremarkably after 10 days without any complaints(fig 6) 


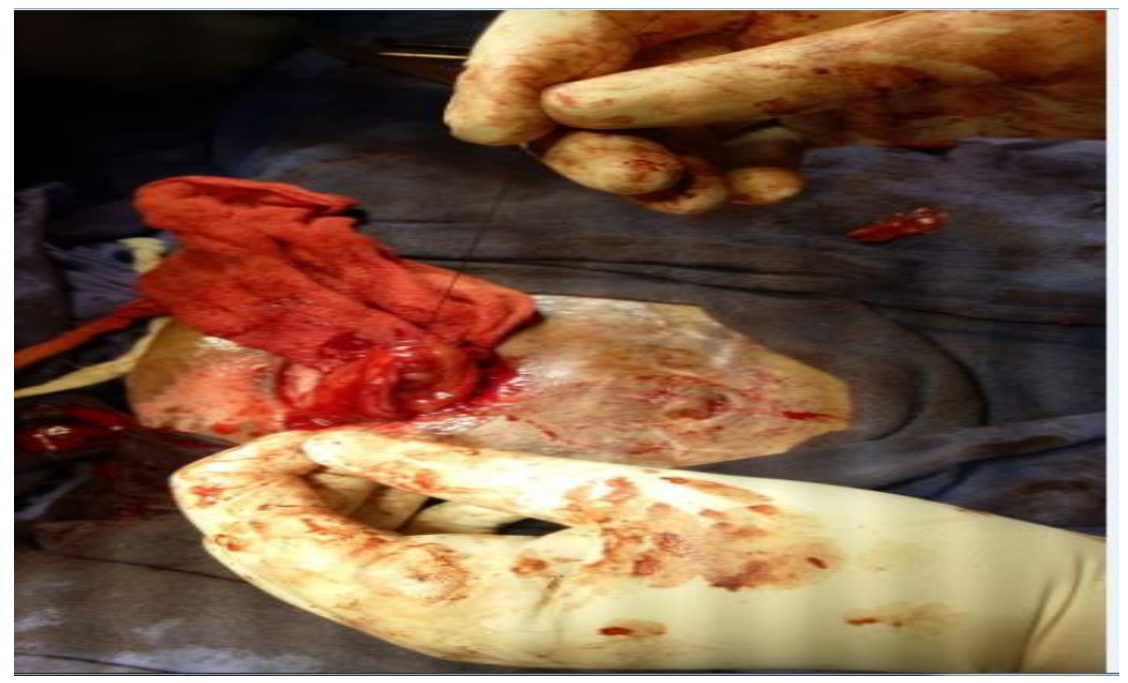

Figure 5 showing single layer closure of double barrel jejunostomy

Patient on follow up after 1 month, on examination vitals was stable , abdomen soft ,suture line healthy and no discharge and other systemic examination being normal .

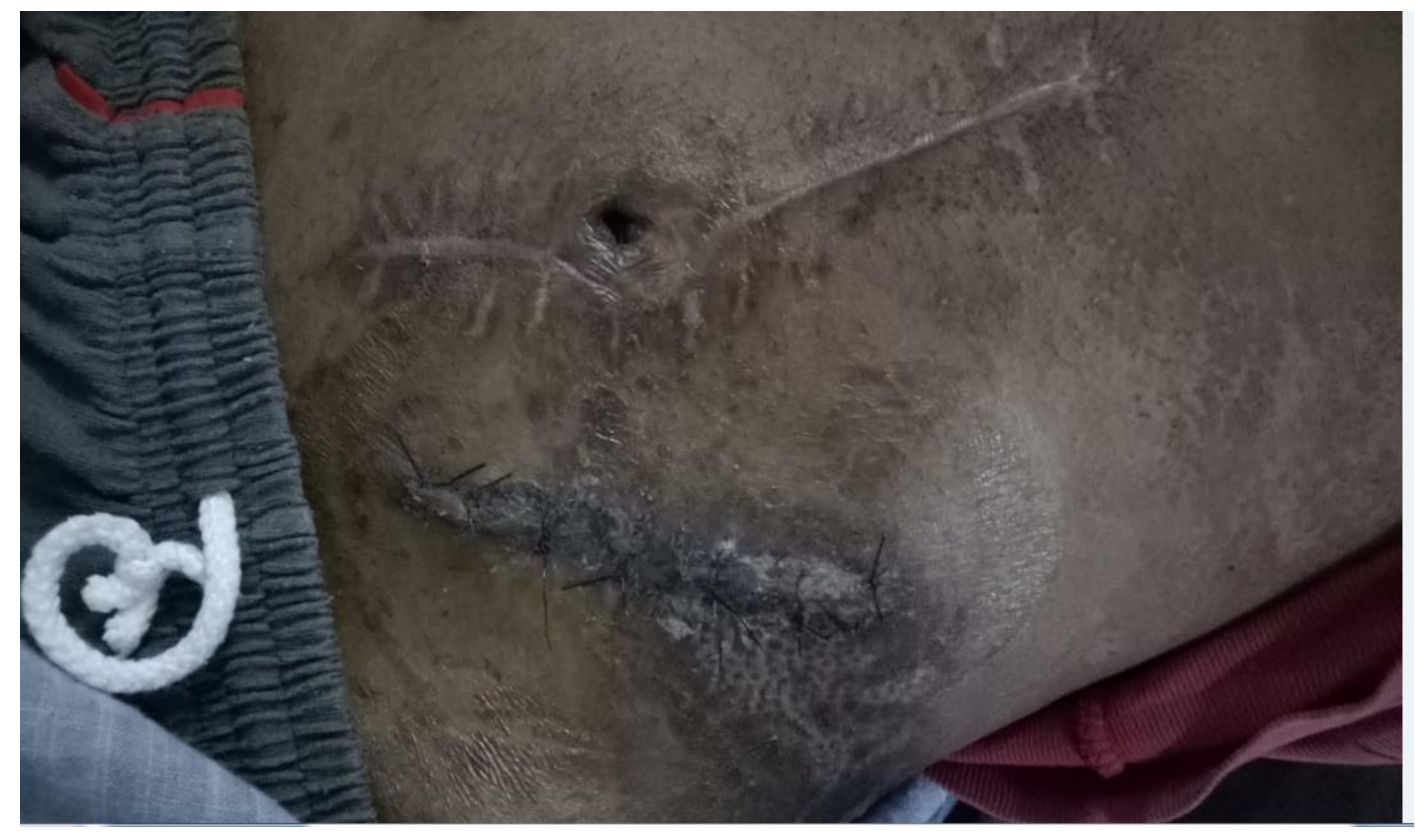

Figure 6: showing healthy suture line

\section{RESULTS AND DISCUSSION}

Bowel injury following blunt abdominal trauma is rare compared to solid organ injury. In a study

by Dauterive A et al,, bowel perforation or mesenteric injury leading to bowel ischemia accounting for $6.9 \%$ of the 870 patients who had laparotomy for blunt trauma during the period of study (1).

According to another study by Watts D et al., perforating small bowel injury accounted for less than $0.3 \%$ of total admissions for blunt abdominal trauma. Complete bowel transection following 
blunt abdominal trauma is rarer(2). In a study by Pikoulis E et al., bowel transection occurred in $2.56 \%$ of 64 patients with blunt intestinal or mesenteric injury [3]. Very few reports of isolated jejunal transection following blunt abdominal trauma have been published in literature [4].

Transection, that is complete loss of bowel continuity, is the most severe form of bowel injury. In such cases, the mechanism of injury is usually mesenteric laceration due to direct compression, or small bowel rupture due to deceleration injury. This would typically affect fixed segments such as the duodenum, duodeno-jejunal flexure, proximal jejunum and terminal ileum(5,6).

In our case, isolated complete transection of proximal jejunum occurred due to application of a localised blunt force to the central abdomen, which resulted in jejunal transection, presumably by direct compression against the lumbar spine.

Bowel injury necessitates urgent laparotomy, delay in which may result in significant morbidity and mortality due to haemorrhage, peritonitis, or abdominal sepsis.

Once the diagnosis is confirmed open or laparoscopic repair is the first and best line of treatment. In our case a Double-barrel stoma was performed in which Both the proximal and distal limbs may or may not be plicated together but are brought out side-by-side through the same wound like a double barrel gun. The proximal limb discharges billous and flatus, and the distal limb discharges mucus and our patient was discharged unremarkably

Thus Improper diagnosis and delayed decision to operate these cases have significant impact on morbidity as well as on mortality but exact duration of delay is still the topic of controversy. According to available literature, delay of eight hours can result in increased morbidity and mortality [7]. Inspite of significant delay by the patient in presenting to us, immediate measures were taken and emergency laparotomy was done which helped us tide over the difficult situation and hence saved the patient.

\section{CONCLUSION}

Complete small bowel transection is a rare injury to follow blunt abdominal trauma. Our case highlights the importance of earliest clinical diagnosis confirmed by available radiological modality and immediate laprotomy and the success of double barrel jejunostomy in spite of significant delay by the patient in presenting to us, and the outcome proved to be life saving.

\section{REFERENCES}

1. Dauterive A, flancbaum L, Cox E. Blunt Intestinal Trauma. [1] Annals of Surgery. 1985;201(2):198-203. 
2. Watts D, Fakhry S. Incidence of Hollow Viscus Injury in Blunt Trauma: An Analysis [2] from 275,557 Trauma Admissions from the EAST Multi-Institutional Trial. The Journal of Trauma: Injury, Infection, and Critical Care. 2003;54(2):289-94

3. Pikoulis E, et al. Presentation of blunt small intestinal and mesenteric injuries. [3] Annals of the Royal College of Surgeons of England. 2000;82(2):103.

4. Sandiford N, et al. Jejunal transection after blunt abdominal trauma: a report of [4] two cases. Emergency Medicine Journal. 2006;23(10):e55.

5. 1 Neugebauer H, Wallenboeck E, Hungerford M. Seventy cases of injuries of the small intestine caused by blunt abdominal trauma: a retrospective study from 1970 to 1994 . J Trauma 1999;46:116-21.

6. Stevens SL, Maull KI. Small bowel injuries. Surg Clin North Am 1990;70:541-60

7. Fakhry SM, Brownstein M, Watts DD, Baker CC, Oller D. Relatively short [7] diagnostic delays $(<8$ hours) produce morbidity and mortality in blunt small bowel injury: An analysis of time to operative intervention in 198 patients from a multicenter experience. $\mathbf{J}$ Trauma. 2000;48:408-15. 